EOMmUn: Communication et organisation

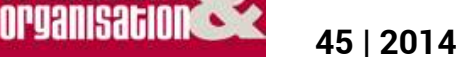

Risques mineurs, changements majeurs

\title{
Dramaturgie du risque
}

Risk staging

Nicole d'Almeida et François Allard

\section{(2) OpenEdition}

Journals

Édition électronique

URL : http://journals.openedition.org/communicationorganisation/4543

DOI : 10.4000/communicationorganisation.4543

ISSN : 1775-3546

\section{Éditeur}

Presses universitaires de Bordeaux

\section{Édition imprimée}

Date de publication : 1 juin 2014

Pagination : 139-152

ISBN : 978-2-86781-904-9

ISSN : 1168-5549

\section{Référence électronique}

Nicole d'Almeida et François Allard, « Dramaturgie du risque », Communication et organisation [En ligne], 45 | 2014, mis en ligne le 01 juin 2017, consulté le 26 avril 2019. URL : http://journals.openedition.org/ communicationorganisation/4543; DOI : 10.4000/communicationorganisation.4543 


\title{
Dramaturgie du risque
}

\author{
Nicole D'Almeida ${ }^{1}$ et Fronçois Allard ${ }^{2}$
}

La question de la visibilité des risques est une question de communication dès lors que la plupart des risques contemporains sont quasiment invisibles à l'œil nu (de l'atome à la bactérie). Il s'agit ici d'analyser les médiations créées et destinées à organiser le couplage du vu et du non vu, du dit et du tacite, du montré et du caché. Ces médiations organisées se déroulent du plus long au plus court : du long texte informatif ou pédagogique mobilisé dans des manuels d'instruction au pictogramme court et dense, indicatif du danger. Dire le risque, c'est autant expliquer qu'alerter. C'est à la fois lisser le risque en l'enveloppant dans un discours qui le prévient et le conjure, c'est aussi le rendre saillant par la présentation d'un visuel dédié dont la complétude et ici interrogée.

Certains travaux se sont déjà penchés sur les contraintes propres à sa représentation qui cherche à rendre présent le non-présent (Couegnas \& Halary , 2002). Des risques mineurs aux risques majeurs, c'est bien là un des enjeux premiers de l'évaluation à la gestion des risques dans une perspective de qualification-formalisation : étant pour la plupart invisibles, les risques ne se rendent visibles qu'au travers de leurs conséquences. Ils doivent donc faire l'objet d'un travail de mise en visibilité, afin de pouvoir être appréhendés, " expertisés », évalués, gérés voire évités.

1 Nicole D'Almeida est chercheure au GRIPIC, porteur du projet Riskcom du CNRS-ISCC, professeur à l'université Paris Sorbonne (Celsa) où elle dirige des formations et des recherches sur les thèmes de la communication organisationnelle-institutionnelle ; Nicole.d'almeida@celsa.paris-sorbonne.fr

Nicole D'Almeida is a Professor at Sorbonne University (Celsa) where she teaches on subjects pertaining to global communications : internal and external communications of corporate and organizational entities, public opinion and public relations, communication of environment and sustainable development, CSR and narrative discourses in these fields.

2 François Allard est Doctorant Contractuel en Sciences de l'Information et de la Communication à l'Université Paris-Sorbonne Celsa, Gripic-ISCC. Ses recherches portent sur la question de la transparence dans les dispositifs sociotechniques d'évaluation et de gestion du risque, en particulier sur l'« Affaire Séralini »; fr.allardhuver@gmail.com

François Allard is a PhD Candidate in Communication Sciences at Paris-Sorbonne University, Celsa, Gripic-ISCC. His researches are focused on the question of transparency in risk evaluation and risk management sociotechnical apparatus, especially on the "Seralini Affair". 


\section{$\mathrm{C} \& O \mathrm{n}^{\circ} 45$}

Nous partirons de l'idée selon laquelle on ne peut dissocier les représentations esthétiques des risques du processus de leur qualification et de leur publicisation. Linscription du risque dans l'espace public découle d'une volonté de rendre visible l'invisible au travers de « configurations de signes de nature différente : langue, écriture, image, forme visuelle, etc. ", comme l'ont montré des travaux précédents sur les rejets polluants (D'Almeida et al., 2005). Plus encore, nous pensons que cette inscription mobilise une symbolique des menaces artificielles ou naturelles qui dépassent l'homme et dont il doit se prémunir par un ensemble de rituels qui mettent à distance le familier et ont des vertus propédeutiques.

Les choix esthétiques, linguistiques et iconiques destinés à configurer et faire circuler le risque sont révélateurs de ce face à quoi une société juge bon de protéger ses membres. Suivant des caractéristiques sociales et historiques propres à l'évolution des enjeux de la communication sur les risques, les signes du risque sont l'affirmation d'un jugement qui entre, dans une société d'énonciation et de dénonciation, dans le cadre d'une communication sur le sensible. Leur livrée graphique révèle, elle, les choix esthétiques et symboliques par le biais, par exemple, de la symbolique des couleurs, mais également par des mécanismes plus anciens qui affirment la puissance sublime du danger, notamment au travers de figures mythologiques. Les signes du risque sont d'une certaine manière des signes " pleins » associés à des problématiques de reconnaissance et d'équipement du risque dans l'espace visible analysé ici dans l'espace domestique, professionnel ou urbain.

Lenjeu est d'identifier ces signes du risque, tout en montrant l'usage qui en est fait dans un cadre de communication dans différents espaces, à travers différentes époques et pour des publics variés. Nous avons ainsi choisi d'observer la construction graphique du risque électrique, du risque lié aux produits chimiques, puis la construction graphique, explicative et politique du " risque requin » dans l'espace réunionnais. La communication sur le risque mobilise une esthétique, elle ne se limite pas à un tout informationnel mais emploie d'autres ressorts graphiques, textuels mais également symboliques voire mythiques.

\section{Petite mythologie du risque}

L'exemple suivant fait apparaître les liens profonds qui existent entre les signes du risque et les enjeux culturels de sa mise en visibilité. Ce premier exemple est un panneau d'avertissement qui rappelle au public et aux usagers de la SNCF le danger qu'il existe à toucher les fils électriques de caténaires, même tombés au sol. Panneau inscrit dans l'espace public à partir des années 1950 et les grandes campagnes d'électrification du réseau ferroviaire français d'après-guerre, il s'agit d'observer un signe du risque fort et faible, fort par sa puissance suggestive, faible car « acclimaté » et devenu presque invisible au regard habitué du public. 
Le panneau (Figure 1) représente un faisceau d'éclairs qui rappelle fortement le faisceau d'éclairs jupitériens présent dans de nombreuses représentations du maître de l'Olympe.
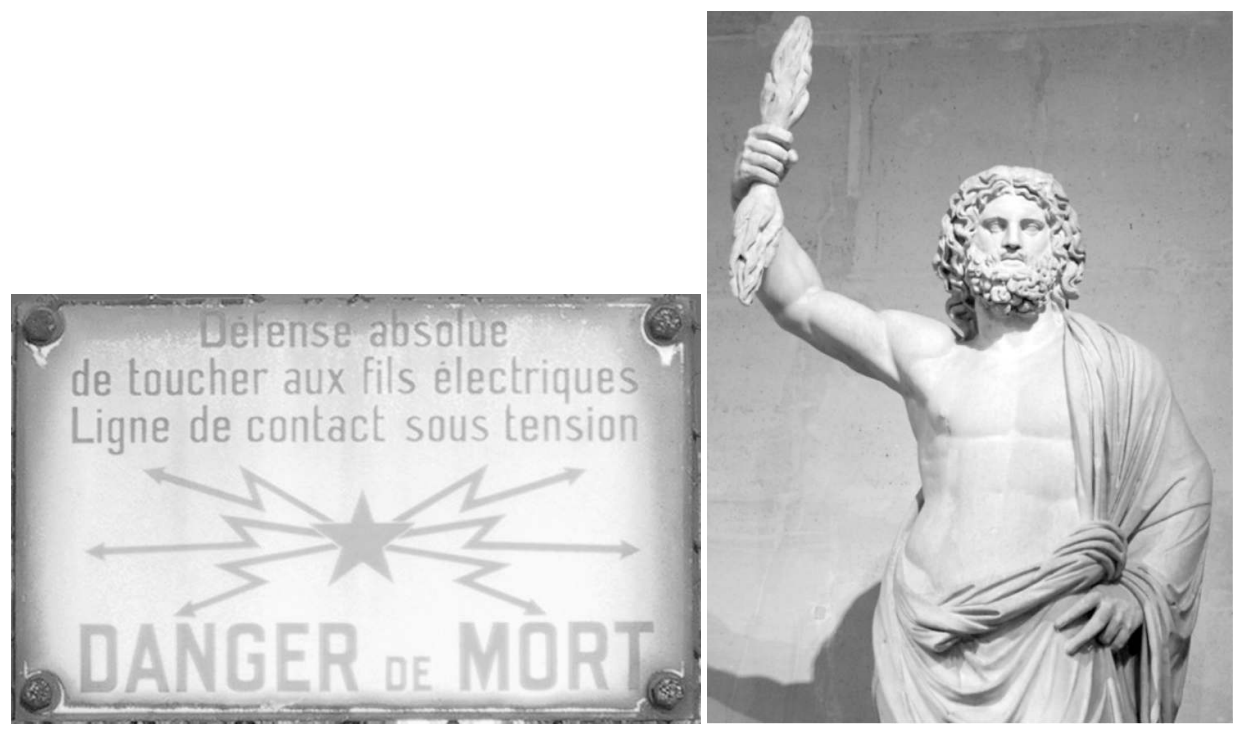

Figure 1 : Panneau danger de mort et Jupiter brandissant les éclairs (Musée du Louvre)

Dans la mythologie grecque puis romaine, l'un des attributs de Jupiter est le faisceau d'éclair qu'il tient dans la main. Au nombre de trois, les éclairs jupitériens avaient pour fonction d'avertir, de punir et de provoquer la fin du monde. Au-delà de la symbolique jupitérienne de la puissance, de la menace qui pèse sur celui qui se défie du commandement divin, se substitue ici l'avertissement de la société ferroviaire face au danger de mort, face au risque d'électrocution rendu visible par les éclairs. Si ici, les trois éclairs reprennent ces fonctions en avertissant d'une punition pour celui qui viendrait à toucher les fils, punition qui peut se solder, non par la fin du monde, mais par sa propre fin, l'attribut de la vigilance est présent en filigrane mais glisse de la figure jupitérienne au lecteur du panneau qui à son tour doit faire preuve de vigilance.

Ce panneau crée un équilibre entre la représentation graphique et symbolique du risque et l'utilisation du texte, en tension entre congruité et nécessité d'expliquer le risque. Ce jeu entre les différents modes de transmission de l'information rappelle l'enjeu même de la communication du risque à savoir éduquer à une vision et à un usage du monde. Il s'agit ici de communiquer une réalité invisible et abstraite - le risque d'électrocution - en l'inscrivant dans un registre communicationnel suffisamment précis pour donner à voir le risque tout en frappant l'attention. C'est dans cet équilibre entre graphie, symbole et écriture que se construit le message. Le texte accentue la force du message et 
du risque encouru par celui qui touche les fils, ce « DANGER DE MORT » dont la police et la taille des caractères mettent bien en valeur les termes « Danger » et « Mort » et les associent non pas simplement grammaticalement mais également visuellement : la menace se fait verbe, se fait parole. Si la sentence n'est pas divine, le risque n'en est pas moins mortel.

Inscrit dans l'espace public depuis près d'une cinquantaine d'années, ce panneau semble presque devenu invisible aux yeux du public, il traduit pourtant la nécessité d'informer sur un risque réel bien quoccasionnel que rappellent de dramatiques faits divers.

\section{Produits chimiques, produits dangereux : produits méchants ?}

Depuis le règlement REACH (2007), l'injonction d'information sur les risques propres aux produits chimiques structure l'espace industriel et fait l'objet de campagnes récurrentes de sensibilisation. Envisageons ici comment sont conduites sur ce sujet des stratégies de communication internes en entreprise sachant que les publics visés sont multiples, ont des niveaux d'instructions différents, tous doivent cependant pouvoir comprendre l'information sur le risque.

Les campagnes de communication de l'INRS (L'Institut National de Recherche et de Sécurité) sont axées sur ce double objectif d'informer de manière précise sur les risques encourus tout en conservant une proximité avec les publics divers des entreprises auxquelles les campagnes s'adressent. En 2006, dans un partenariat avec les Caisses Nationale et Régionales d'Assurance Maladie, l'INRS produit une brochure intitulée « Moi dans mon entreprise, j'étiquette les produits dangereux $»^{3}$ et une nouvelle brochure en 2013, à l'occasion de la mise en place d'un nouveau référentiel d'étiquetage des risques dans le cadre du règlement européen "Classification, Labelling and Packaging " directement hérité de REACH : "Étiquettes de produits chimiques. Attention, ça change !». Ces deux communications ont pour objectif de sensibiliser les publics internes à l'entreprise face aux risques liés à l'utilisation de produits chimiques mais également de les former à la lecture et l'utilisation des étiquettes propres à chaque produit chimique. C'est le message directement apposé sur le produit qui fait montre de la visibilité du risque dans sa proximité et donc dans sa potentialité destructive. Dans ce document, les signes du risque choisis associent représentations officielles et réglementaires du risque à une représentation symbolique et humoristique, voire d'un certain paternalisme dans le ton qui témoigne de choix communicationnels particuliers. Ces choix nous invitent à poser la question de la difficulté à communiquer sur le risque de manière verbale et esthétique voire éthique. Est ici posée la question de la concurrence des visuels, des textes et des tons, de la concurrence des jugements, l'enjeu de la mise en visibilité du risque étant aussi celui de la «captation non seulement verbale mais aussi sensorielle »

3 Dépliant INRS ED 745, Édition INRS, décembre 2004. 
de l'attention du lecteur sur le signe du risque qui réalise alors un « désir de présence dans les lieux et les esprits, occupation somptueuse ou discrète de l'espace » (D'Almeida, 2007).

De prime abord, le texte et le choix du vocabulaire utilisés dans cette publication interpellent. Le choix de l'adjectif "méchant » caractérisant les produits chimiques est un choix assez particulier de vulgarisation qui associe puis substitue des groupes nominaux dans le texte à destination des publics internes : " produits chimiques " est remplacé par " produits dangereux ", puis " produits dangereux " par " produits méchants " créant là une série superordonnée fonctionnant par syllogisme (Jacobi, 1990). L'emploi du terme "méchant ", découle d'une volonté de renforcer l'attention envers ces risques mineurs en procédant à leur banalisation, leur normalisation, et en somme leur prolifération afin de développer l'apprentissages de pratiques, de comportement et d'habitudes qui ont une vertu prophylaxique face au risque. Le dispositif des signes du risque est donc à la fois un dispositif au sens foucaldien, c'est-à-dire qu'il produit un sujet qui doit être un sujet réfléchi, expert de soi et du risque ; c'est également un dispositif au sens deleuzien en ce qu'il produit des lignes de visibilité du risque. Analysons le choix du dragon comme principal vecteur graphique et symbolique du risque.

\section{MOI, DANS MON ENTREPRISE}

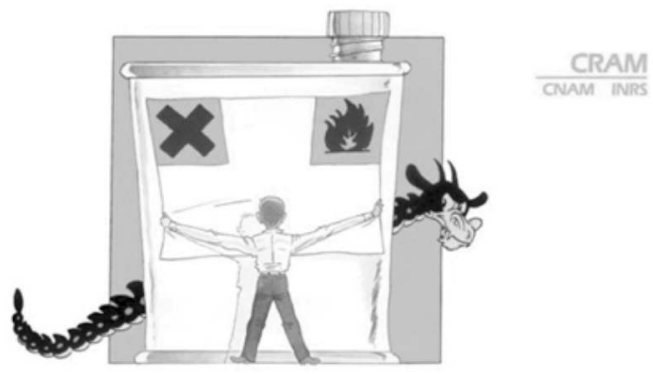

\section{J'ETIQUETTE LES PRODUITS MECHANTS}

Figure 2 : "Moi, dans mon entreprise j'étiquette les produits méchants » (Dépliant INRS)

Dans ce visuel et dans l'ensemble du dépliant, le principal élément représentant le risque est le dragon, mais également les pictogrammes du risque (dans la version précédant la nouvelle réglementation CLP de 2009) représenté directement mais également indirectement par la prédominance de la teinte orangée, cette couleur la moins aimée de la société occidentale (Pastoureau). Le dessin du dragon décliné sous toutes ces formes et ayant différents attributs, différentes nocivités, représente le risque propre à chaque produit chimique. Ainsi, non seulement le dragon se cache-t-il derrière 
chaque produit (Figure 2), ce qui témoigne de sa dangerosité mais il perce également la main (corrosion) ou pénètre dans les voies respiratoires (souffle) et peut les ronger (acide) sans compter le risque d'explosion ou de feu qu'il faut maîtriser comme on " apprivoise " le dragon.

Le dragon fait partie des animaux mythiques que l'on associe aux forces chtoniennes, forces naturelles dévastatrices mais également sources de pouvoir pour qui sait les vaincre ou les contrôler. Une tâche qui échoit en premier aux figures religieuses, avec Saint-Georges et Saint-Clément, à qui est confiée métaphoriquement (comme aujourd'hui aux scientifiques) la mission de lutter contre le « dragon ». Rappelons également que le mot dragon est issu du verbe grec drakein qui signifie « observer avec un regard perçant ». À l'attribut de la puissance s'adjoint la vigilance et l'omniprésence du regard, comme le dragon Smaug qui surveille le trésor de la Montagne solitaire dans Bilbo le Hobbit.

A contrario, la brochure de 2013, "Etiquettes de produits chimiques. Attention, ça change ! ", délaisse complètement le registre de l'humour et de la symbolique mythologique pour une communication sobre basée sur l'évolution du signe (Figure 3). La livrée graphique du signe du risque évolue dans la forme, passant du carré au losange mais également de l'orange au rouge. Dans cette brochure, point de dragon ni de figure mythique pour illustrer la menace invisible des produits chimiques. C'est la dramaturgie des seuls symboles sur le risque qui se substitue à ces représentations précédentes : les pictogrammes sont plus vifs, par la couleur rouge, plus saillants, par les arêtes du losange, et en même temps plus tragiques, avec l'apparition d'un signe portant la menace du cancer et des mutations d'ordre tératogénique. On remarque par ailleurs que la présence de l'individu sur les représentations diminue : de celui qui subit les attaques du dragon en 2006 et peut le contrôler en étiquetant les produits, on passe à celui qui se contente d'apposer la marque du changement sur les produits, silhouette noire en filigrane.

Dans cette brochure de 2013, on constate leur importance et le choix de les agencer dans des tableaux comparatifs et explicatifs, faisant des signes la portion congrue de la mise en visibilité du risque. En effet, comme le précise de manière insistante la brochure, dans un cartouche en forme de losange rouge : «Attention! Certains dangers ne sont pas symbolisés par un pictogramme. C'est pourquoi il est très important de lire entièrement l'étiquette ». Le signe s'efface devant le texte qui le supplée et qui lui seul peut verbaliser la complexité du danger. D'autre part, le ton adopté et les choix en termes de présentation, font montre d'une volonté de privilégier l'information par rapport à la démarche pédagogique précédente, infantilisante et humoristique, remplacée ici par une information simplifiée mais purement factuelle, mobilisant un autre paradigme de la communication. 


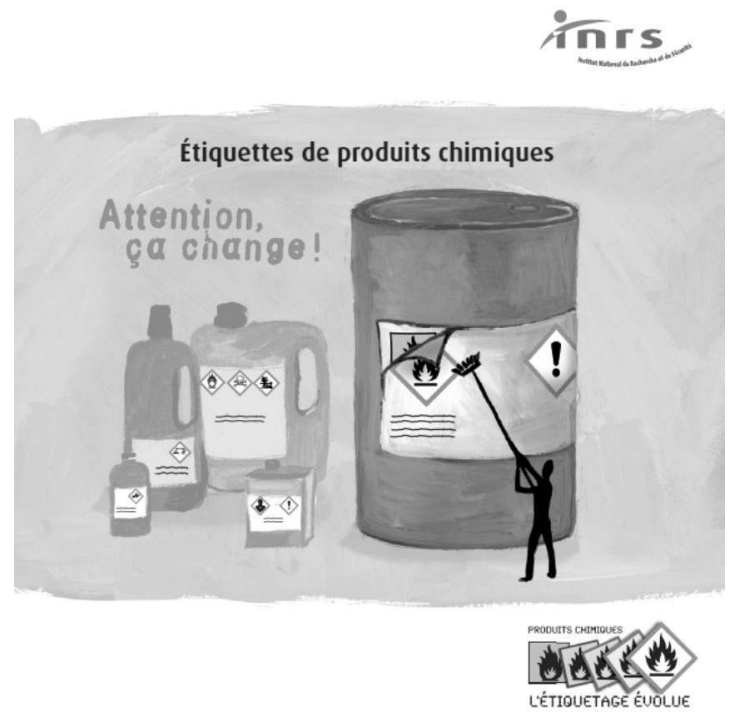

Figure 3 : Brochure INRS, Étiquettes de produits chimiques. Attention, ça change !

L'analyse des signes du risque dans cette entreprise allie donc des éléments symboliques et graphiques « intemporels » à des enjeux propres à un contexte communicationnel particulier. Ainsi, les signes choisis pour le risque associent la symbolique populaire à la représentation officielle, tout en choisissant de "dédramatiser " le risque sur le ton de l'humour avec un vocabulaire plus familier. L'évolution réglementaire est par contre l'occasion d'une évolution de ton : on passe de l'humour à l'injonction, et plus exactement à l'injonction du lire face au voir, symbole à ce niveau d'une forme de logocentrisme face au symbolisme et à la mise en images de risques invisibles. Ces choix témoignent d'une tension entre visuel et textuel dans l'obligation d'informer.

\section{Le risque requin à La Réunion : un autre scénario pour "Les Dents de la Mer"}

Envisageons à présent le cas de ce qui en 2011 va être nommé le « risquerequin » sur l'île de La Réunion, située en plein Océan Indien.

Les requins sont partie intégrante de certains océans et leur présence est en ce sens « naturelle ", conforme à l'ordre de la nature. L'absence ou la disparition de requins pourrait, si cela se produisait, générer un débat ou une réglementation comme c'est le cas pour les baleines. Ici se produit une situation inverse et c'est la présence de requins qui va devenir un problème public et va constituer la trame d'un travail social de construction d'une nouvelle figure du risque. Plusieurs requins se sont en effet approchés des côtes et ont attaqué des surfeurs au cours de l'été 2012, provoquant la mort de deux d'entre eux, et portant à un total de huit les attaques ayant eu lieu en moins de vingt mois. Cette histoire n'est pas le remake des Dents de la Mer, film inquiétant 
mais adulé qui met en scène la puissance de la nature et la menace qu'elle fait peser sur des populations trop confiantes à l'égard du progrès et de la maîtrise supposée des forces naturelles. Si ce film hollywoodien nous rappelle la fragilité de notre condition tout en mettant à distance le danger, le traitement réel d'une situation réelle nous fait découvrir un tout autre scénario marqué par le passage du plus court au plus long, du local au national, de l'information à la communication et du médiatique à l'institutionnel.

La présence avérée mais invisible de requins va être mise en visibilité par des accidents mortels, drames qui constituent la trame d'une activité collective de jugement, de réflexion, de propositions et d'élaboration de signes de ce danger. Un récit collectif va se construire à plusieurs voix autour d'un mystère : «le mystère de l'augmentation des attaques de requins ", titre d'un article du journal Le Monde du 26 août 2011. Ce mystère va être conjuré, éclairci par un travail d'information (élaboration de signes et pictogrammes) puis de communication (débat public) faisant d'un risque mineur mais avéré le centre d'une attention importante. Tous les ingrédients d'une situation de crise et de création d'un récit mythologique au sens barthésien sont ici réunis. L'imaginaire fantasmagorique des monstres marins est réactivé dans un espace insulaire où se sont imposés des regroupements et modes de vie urbains souvent oublieux des forces de l'environnement. Le poids des médiacultures et le souvenir cinématographique des Dents de la Mer se ravivent alors. La place des sports de glisse et la culture du surf avec son cortège d'euphorie et de fluidité se heurtent brutalement au principe de réalité lorsque l'exigence contemporaine de sécurité totale est prise en défaut. Enfin, les enjeux économiques liés au tourisme sont menacés par la présence insistante des requins. Trois peurs se conjuguent à ce moment lié au surgissement d'un passé oublié : le retour des forces de la nature dans une société urbanisée, le retour d'un souvenir cinématographique dans un cadre non hollywoodien, le retour à l'isolement ancestral et à la pénurie dès lors que les échanges économiques sont menacés. Ce drame potentiel va constituer la trame d'un travail collectif d'exploration, de discussion, de création de signes adéquats installés dans l'espace public des villes et des plages concernées et de débats nombreux. Le travail d'information et de prévention qui prend la forme de création de pictogrammes n'est pas une réponse isolée, de type technocratique ou technique, il est enchâssé dans un travail de mise en débat public. Élaborer les signes du risque-requin et donner à voir l'invisible et le danger potentiel ne suffit pas : les choix graphiques vont être imbriqués dans des choix politiques où sont enrôlées de nombreuses catégories d'acteurs.

Les premiers signes du risque prennent la forme de panneaux (qui seront ensuite complexifiés) dans lesquels apparaissent deux caractéristiques intéressantes : la tension des couleurs entre rouge et bleu, la tension entre la surface des pictogrammes et celle du texte. Les premiers panneaux font apparaître des pictogrammes répondant aux normes AFNOR (Agence 
Française de Normalisation) et aux caractéristiques du genre que sont la sélection d'un élément (l'aileron du requin) intégré dans une structure stéréotypée cohérente (Bordon, 2004). Le choix de la couleur rouge exprime le danger et l'interdit, il est ici allié au bleu pacifique de l'élément marin. S'il est vrai comme l'a montré Michel Pastoureau que les couleurs n'ont de sens que par opposition et contraste les unes par rapport aux autres, la complémentarité-opposition du bleu et du rouge évoque la tension du voir et du savoir, de l'acquis et du donné, du civilisationnel et du naturel. Les pictogrammes, signes minimalistes et universels sont ici accompagnés et complétés par des messages écrits, minimalistes dans un cas, plus développés dans l'autre. De l'annonce d'une « présence » dans le premier cas, nous passons à l'annonce d'un « danger» dans le second cas (Figure 4).

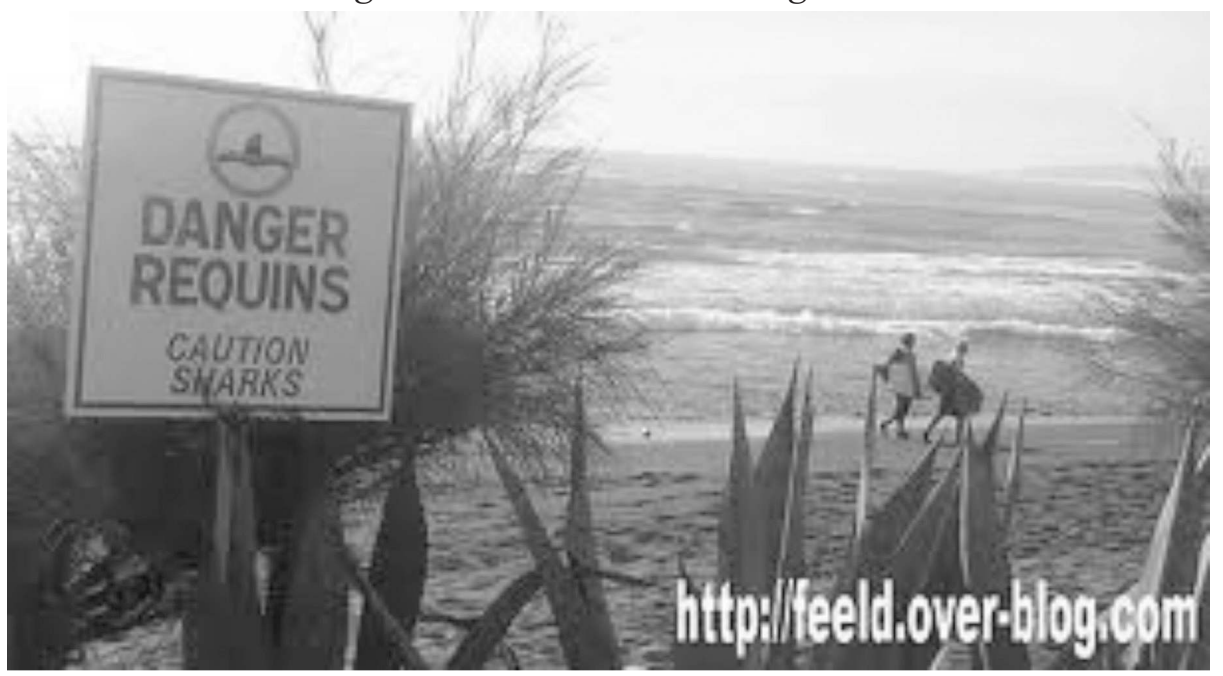

Figure 4 : Affichage danger requin

Un nouvel accident provoquant la mort d'un champion de surf ravive l'émoi et suscite la mise en avant d'un signe unique : le drapeau rouge signifiant l'arrêt de toute activité nautique sur l'île. Ce signe minimaliste mais puissant est alors installé sur les plages tandis que se développe le débat public d'abord de manière spontanée puis organisée. Le mystère rebondit, s'amplifie et génère une conversation généralisée à tous niveaux et l'histoire locale, située, va sortir du territoire et être relayée par un sensationnalisme morbide qui transforme le fait divers en un fait de société que traitent médias traditionnels et médias nouveaux (réseaux sociaux, blogs) en France et en Europe. La mise en visibilité d'un risque jusqu'alors invisible devient mise en médias et mise en débat. La médiatisation de ces faits divers s'accompagne d'une réflexivité et d'une prise de conscience que relaient les maires et députés des villes concernées, organisateurs d'une concertation publique. Des ateliers sont mis en place pour réfléchir aux systèmes d'alerte et à une réglementation, 
à la gestion du risque et au lancement d'une étude scientifique. Il est parallèlement fait appel au travail scientifique qui prend alors plusieurs formes : opération de prélèvement de requins, lancement du programme CHARC (Connaissance de l'Habitat des Requins Côtiers), mobilisation d'experts et marquage de requins afin de comprendre leurs circuits de déplacement. On assiste ici à la construction de l'expertise du risque à tous les niveaux. L'enrôlement des populations sur ce sujet est massif mais il ne converge pas vers une position unifiée et suscite des oppositions entre groupes. Les enjeux de vie (loisir des jeunes), de survie (professionnels du tourisme) et de sécurité (mission des pouvoirs publics) s'entrechoquent. Les commerçants de l'île lancent un appel à manifester pour marquer leur inquiétude face aux répercussions de ce qu'ils considèrent comme une dramatisation médiatique d'un risque mineur. Les institutions de la République sont interpellées : un comité réunionnais de réduction du risque requin est créé ainsi qu'un fonds d'aide et de subvention. Le milieu associatif n'est pas en reste : l'association Prévention Requin Réunion est créée tandis que Brigitte Bardot intervient auprès du préfet de l'île pour demander « de quel droit nous permettons-nous d'envahir tous les milieux en faisant le ménage de tout ce qui peut représenter un danger pour l'être humain ?".

À ce travail collectif succède l'élaboration d'un panneau de grande taille partagé entre le rouge du danger et le bleu du conseil, visant par la taille importante du texte une perspective d'information-éducation des publics et allant au-delà de l'injonction stricte et muette du pictogramme (Figure 5a).

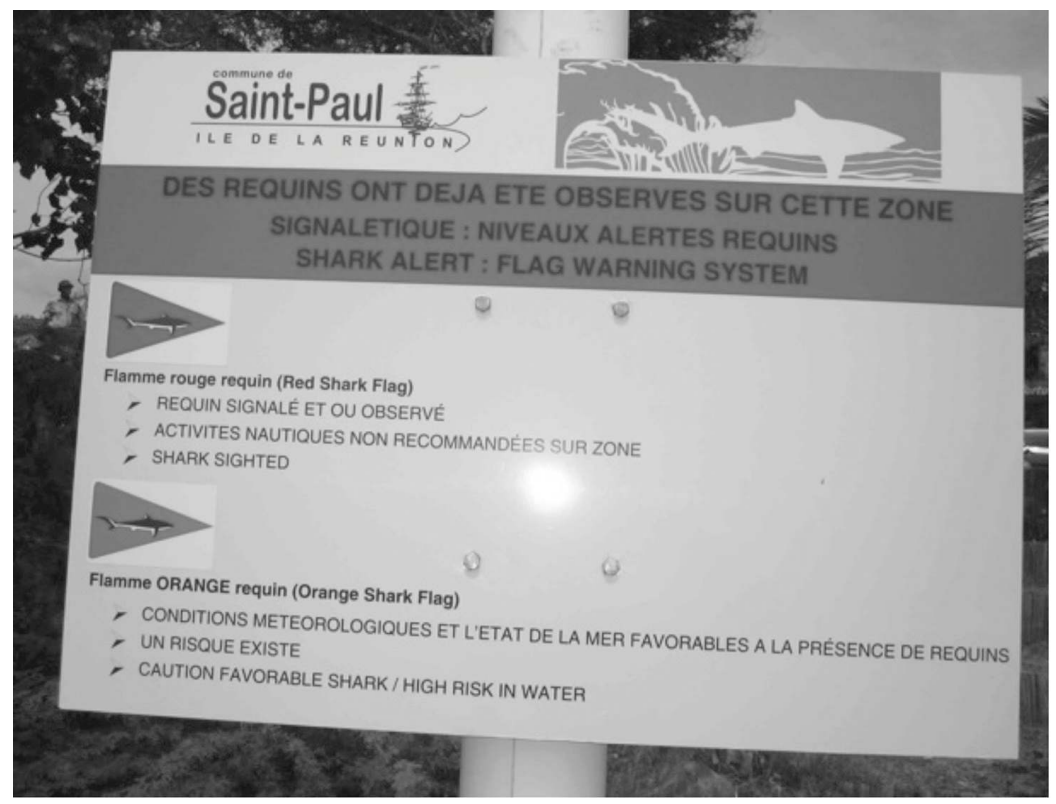

Figure $5 a$ : Panneau sur le « risque requin » 
L'argument d'autorité qu'incarne le seul pictogramme ne suffit pas, il est complété par un texte long et didactique qui présente un état des savoirs et des conseils de prudence. Entre le format court et le format long des panneaux conçus pour informer du danger s'intercale un apprentissage collectif et une maîtrise partagée et discutée de cette nouvelle forme de risque (Figure 5b).

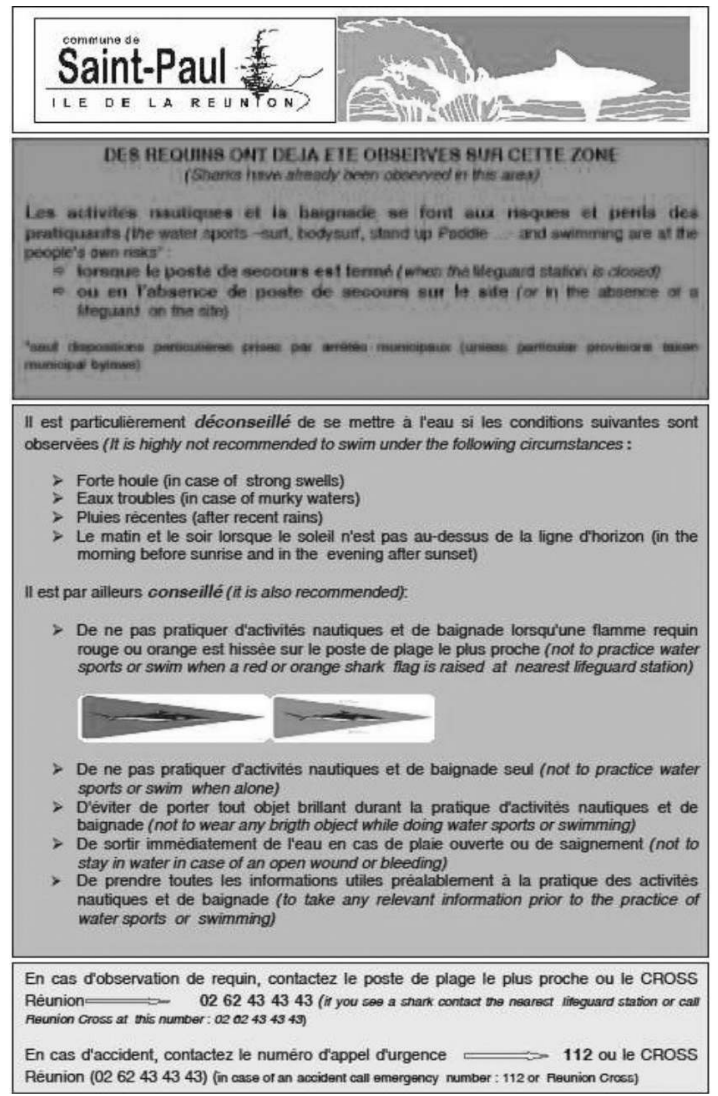

Figure $5 b$ : Panneau sur le « risque requin »

Ces panneaux révèlent en creux une véritable politique du signe, les choix graphiques étant animés par des choix politiques, par l'existence d'un débat public particulièrement vif.

Mais, un signe peut en cacher un autre! La thématisation en commun du risque-requin sur l'île de La Réunion a ravivé le dialogue des habitants, il a généré conflits et accords et, ce faisant, il a développé du politique dans une question qui ne l'est pas a priori. Le lancement en 2012 de la campagne institutionnelle «Bonne nouvelle de La Réunion » est une modalité de sortie de crise qui opère un effet de pacification et d'unification d'un lieu menacé dans ses échanges. Cette campagne met sous le boisseau le risque requin par la 
mise en avant d'un autre élément marin, positif cette fois (la baleine), destiné à rassurer les populations-cibles qui liront ce message dans le métro parisien faisant ainsi reculer le requin des esprits. La campagne " Même pas peur » de l'entreprise Pardon est une tentative de dédramatisation mais qu'assurera de manière plus durable une campagne publicité misant sur la randonnée ou le farniente en bord de plage...

\section{Éveiller la paresse du signe}

À travers les exemples convoqués, il apparaît bien que la dimension symbolique et muette des signes et pictogrammes est à la fois puissante et insuffisante : la force des symboles suppose le verbe et appelle une discussion, un débat. La puissance suggestive des pictogrammes campe un horizon muet du sens commun qui, on le voit, demande toujours à être activé, explicité et discuté. Inventer des médiations destinées à accompagner un parcours qui rend visible le risque invisible, qui " présentifie » le danger ne peut se faire uniquement sur le mode visuel. Le donner à voir s'accompagne nécessairement d'un donner à lire, à penser et à dire. Le visible suppose un dicible.

La question de la dualité entre visible / invisible, écrit / graphique et communication symbolique / information factuelle invite à repenser ici l'idée de pharmakon telle que présentée par Platon (Phèdre) puis reprise par J. Derrida (1972, p. 365) : «Le pharmakon est ce qui, surgissant du dehors force le vivant à avoir rapport à son autre, au risque d'un mal d' "allergie" ». En effet, les signes du risque présentent en premier lieu des éléments qui s'apparentent à ce pharmakon-poison évoqué par Platon, c'est-à-dire l'oubli, la paresse et le signe qui ne peut pas parler pour lui-même. Le pharmakon poison (signe assoupi qui occulte et n'indique plus) doit être contrebalancé par le pharmakon remède qui vivifie le sens. La concurrence entre la logique textuelle, informative et l'ordre symbolique et graphique s'inscrit dans cette tradition de la lutte entre écriture et oralité propre à notre civilisation logocentrée. Le choix de signes graphiques suscite une réflexion sur la manière de communiquer le risque dans l'espace public, sur la symbolique qu'on peut associer aux risques et sur sa limite : pharmakon au sens double le risque est toujours le résultat d'une négociation, d'un défi entre des forces antagonistes, entre sa présentation et son assimilation, entre sa maîtrise et l'impossibilité de pouvoir l'intégrer totalement.

Dès lors, il nous faut rappeler que le signe du risque n'existe que dans un contexte sémio-politique particulier, celui de la société qui lui attribue du sens au travers d'un travail de mise à distance et d'usage public de la raison. La dimension symbolique et muette des signes et pictogrammes est à la fois puissante et insuffisante : l'ambivalence des symboles appelle une discussion, un débat, la construction explicite de significations sociales qui étendent l'horizon muet du sens commun qui demande toujours à être réactivé, explicité et discuté. 


\section{$\mathrm{C} \& O \mathrm{n}^{\circ} 45$}

\section{BIBLIOGRAPHIE}

ALLARD François, Transformations et circulations de la notion de "risque » au sein de la Commission Européenne, Master Recherche, CELSA, 2011, 115 p.

BARTHES Roland, Mythologies, Seuil, 2010 [1957], 252 p.

BORDON Emmanuelle, L'interprétation des pictogrammes, L'Harmattan, 2004, 324 p. COUÉGNAS Nicolas, Marie-Pierre HALARY, « Signification et communication du risque : le cas du nucléaire ", in Quaderni, n 48, 2002, p. 89-99.

D'ALMEIDA Nicole, La société du jugement: essai sur les nouveaux pouvoirs de l'opinion, Armand Colin, 2007, 252 p.

DERRIDA Jacques, La dissémination, Seuil, 1972, p. 126.

DUCHET-SUCHAUX Gaston, PASTOUREAU Michel, Le bestiaire médiéval : dictionnaire historique et bibliographique, Paris, La licorne d'or, 2002, 167 p.

GILBERT Claude "La fabrique des risques ", Cabiers internationaux de sociologie, $n^{\circ} 114,2003$, p. 55-72.

GOODY Jack, "Oralité et modernité dans les organisations bureaucratiques ", Communication E langages, $\mathrm{n}^{\circ} 136,2003$, p. 4-12.

JACOBI Daniel, "Les séries superordonnées dans les discours de vulgarisation scientifique », Langages, $n^{\circ}$ 98, 1990, p. 103-114.

PASTOUREAU Michel, Dictionnaire des couleurs de notre temps : symbolique et société, Bonneton, Paris, 1999, 255 p.

Résumé : Face à la multiplication des risques et à leur normalisation, la question de leur mise en visibilité et de leur mise en discours est un enjeu pour les organisations et les institutions à la poursuite d'un illusoire "risque zéro ». Montrer, dire, identifier, inscrire et prévenir les risques sont autant d'actions qui appartiennent à un registre communicationnel distinctif. Ces actions sont le reflet de médiations organisées, prises en charge par des organisations diverses au sein d'espaces et de temporalités spécifiques, allant de l'espace public en général, à des espaces particuliers comme l'espace professionnel voire touristique. Nous analysons ici, dans une petite mythologie contemporaine du risque, trois mises en signes diachroniques du risque : le risque électrique puis le risque chimique et enfin, le « risque requin ».

Mots-clés : risque, signe, discours, sémiologie, chimie, électricité, requin.

Abstract: Facing risks' proliferation and standardization, the question of their visibility and their expression is a challenge for organizations and institutions seeking a fanciful "zero risk". Showing, telling, identifying, inscribing and preventing risks are all actions that belong to a distinctive communicative range of activities. These actions reflect the mediations held and supported by various organizations, and within specific areas and time frames ranging from public space in general to specific areas such as professional or tourist areas. We analyze here, in a small contemporary risk mythology, three diachronic sets of risk signs: the electrical risk, the chemical hazards, and finally the "shark risk".

Keywords: risk, sign, speech, semiology, chemistry, electricity, shark. 
